\title{
Nonaxisymmetric stability in the shearing sheet approximation
}

\author{
A. Brandenburg ${ }^{1}$ and B. Dintrans ${ }^{2}$
}

\author{
1 NORDITA, Blegdamsvej 17, 2100 Copenhagen $\emptyset$, Denmark \\ e-mail: brandenb@nordita.dk \\ 2 Observatoire Midi-Pyrénées, CNRS et Université Toulouse 3, 14 avenue Edouard Belin, 31400 Toulouse, France
}

Received 4 December 2005 / Accepted 6 January 2006

\begin{abstract}
Aims. To quantify the transient growth of nonaxisymmetric perturbations in unstratified magnetized and stratified non-magnetized rotating linear shear flows in the shearing sheet approximation of accretion disc flows.

Methods. The Rayleigh quotient in modal approaches for the linearized equations (with time-dependent wavenumber) and the amplitudes from direct shearing sheet simulations using a finite difference code are compared.

Results. Both approaches agree in their predicted growth behavior. The magneto-rotational instability for axisymmetric and non-axisymmetric perturbations is shown to have the same dependence of the (instantaneous) growth rate on the wavenumber along the magnetic field, but in the nonaxisymmetric case the growth is only transient. However, a meaningful dependence of the Rayleigh quotient on the radial wavenumber is obtained. While in the magnetized case the total amplification factor can be several orders of magnitude, it is only of order ten or less in the nonmagnetic case. Stratification is shown to have a stabilizing effect. In the present case of shearing-periodic boundaries the (local) strato-rotational instability seems to be absent.
\end{abstract}

Key words. accretion, accretion disks - hydrodynamics - instabilities - magnetohydrodynamics (MHD)

\section{Introduction}

The gas in accretion discs is generally though to be turbulent (Shakura \& Sunyaev 1973). This allows potential energy to be converted into kinetic energy which can then be dissipated (e.g., Frank et al. 1992). This in turn can lead to significant amounts of observable radiation. To sustain the turbulence, there has to be some instability. This instability is now generally thought to be the Balbus-Hawley or magneto-rotational instability (hereafter referred to as MRI; see Balbus \& Hawley 1998 for a review). This instability is linear and local in that it does not rely on the presence of boundaries. It exists already in the axisymmetric case in the presence of an external vertical field, which makes this instability technically easy to study.

In the nonaxisymmetric case, an azimuthal pattern will be sheared out differentially, i.e. patterns in the inner parts of the disc are advected faster than in the outer parts. After an increasing number of orbits, this causes more and more rapid variations in the radial direction. Loosely speaking, this makes the radial wavenumber time-dependent.

In the presence of radial boundaries or other radial nonuniformities, and certainly also in the presence of nonlinearity, any radial pattern that was initially harmonic will become anharmonic. This produces a spectrum of wavenumbers even for monochromatic initial conditions. Mathematically, the stability of such a problem can be studied by solving a one-dimensional eigenvalue problem subject to radial boundary conditions (Ogilvie \& Pringle 1996). Even though boundary conditions are important in that case, the results may still be relevant for driving turbulence provided the instability is not limited to the vicinity of the boundaries.

In many numerical simulations of accretion disc turbulence the shearing sheet (or shearing box) approximation has been employed (Hawley et al. 1995; Matsumoto \& Tajima 1995; Brandenburg et al. 1995). This approximation represents the other extreme, where boundaries and any non-uniformities are strictly removed. Any instability that survives under these conditions is often referred to as a "local" instability, even though its onset properties may depend on the system size (as is typical of all long-wavelength instabilities).

For nonaxisymmetric solutions, a purely analytic treatment of the shearing sheet model is generally impossible, because the solutions exhibit a complicated temporal behavior that cannot even be approximated by an exponential time evolution. In fact, all nonaxisymmetric solutions only exhibit transient growth whose speed of growth depends on the instantaneous wavevector. However, for the MRI it turns out that the Rayleigh quotient obtained from the time-dependent nonaxisymmetric 
solution is a good approximation to the usual eigenvalue in the much simpler axisymmetric problem. The purpose of the present paper is to attempt a more systematic survey of the nonaxisymmetric problem by studying the dependence of Rayleigh quotient in different two-dimensional parameter planes. We begin with the fairly well understood MRI and then turn to the less well understood problem with vertical density stratification, but no magnetic field. The latter case is less well understood, although it has been shown that in the presence of radial boundaries there is a linear instability of the Taylor-Couette problem with density stratification along the axis (Molemaker et al. 2001; Shalybkov \& Rüdiger 2005; Umurhan 2005). This "strato-rotational" instability (hereafter refereed to as SRI) has been confirmed numerically in the presence of boundaries (Brandenburg \& Rüdiger 2006), but it may still be local in character, i.e. its properties may not be sensitive to the presence of boundaries (Dubrulle et al. 2005).

It turns out that in the nonaxisymmetric case without boundaries there can at most only be transient growth. This is also true of the MRI, which has an instantaneous growth rate quite analogous to that in the axisymmetric case. In shearing sheet simulations, sustained instability can only be the result of nonlinearity allowing mode coupling and hence the recycling of energy into new modes viable of repeated growth.

\section{The shearing sheet formalism}

The full hydrodynamic and magnetohydrodynamic equations can always be written in the form

$$
\frac{\mathrm{D} \boldsymbol{q}}{\mathrm{D} t}=\boldsymbol{F}(\boldsymbol{q})
$$

where $\boldsymbol{q}$ is a state vector combining all components of velocity, density, entropy, and the magnetic field, and $\mathrm{D} / \mathrm{D} t=\partial / \partial t+\boldsymbol{U} \cdot \boldsymbol{\nabla}$ is the advective derivative. The velocity $\boldsymbol{U}$ can be subdivided into an equilibrium solution (the mean flow $\bar{U}$ ) and the departures from the mean flow, so

$$
\boldsymbol{U}=\bar{U}+\boldsymbol{u}
$$

In the shearing sheet approximation, the mean flow depends linearly on the cross-stream coordinate, say $x$, so we assume the mean flow to be

$\overline{\boldsymbol{U}}=(0, S x, 0)^{T}$,

where $S$ denotes the gradient of the shear flow. In a local model of a keplerian disc we have $S=-\frac{3}{2} \Omega$, where $\Omega$ is the local angular velocity.

Inserting Eq. (3) in Eq. (1) yields

$\frac{\partial \boldsymbol{q}}{\partial t}+S x \frac{\partial \boldsymbol{q}}{\partial y}+\boldsymbol{u} \cdot \boldsymbol{\nabla} \boldsymbol{q}=\boldsymbol{F}(\boldsymbol{q})$

where the second term on the left hand side has an explicit $x$ dependence. It turns out that none of the other terms have an explicit $x$ dependence. This is because we are restricting ourselves only to linear shear flows, so the flow has constant gradients.
Equation (4) can be linearized with respect to the departures from the equilibrium solution, $\overline{\boldsymbol{q}}$, so we write $\boldsymbol{q}=\overline{\boldsymbol{q}}+\boldsymbol{q}^{\prime}$ and have

$\frac{\partial \boldsymbol{q}^{\prime}}{\partial t}+S x \frac{\partial \boldsymbol{q}^{\prime}}{\partial y}=\mathbf{L} \boldsymbol{q}^{\prime}$

where $\mathbf{L}$ is a matrix with differential operators and constant coefficients. Equation (5) can be solved by making the "shearing sheet" ansatz (Goldreich \& Lynden-Bell 1965; Balbus \& Hawley 1992a)

$\boldsymbol{q}^{\prime}(x, y, z, t)=\hat{\boldsymbol{q}}(t) \exp \left[\mathrm{i} k_{x}(t) x+\mathrm{i} k_{y} y+\mathrm{i} k_{z} z\right]$.

Note that by differentiating Eq. (6) with respect to $t$, one pulls down a term proportional to $\mathrm{i}\left(\mathrm{d} k_{x} / \mathrm{d} t\right) x$. This explicitly $x$-dependent term can be arranged to cancel the second term of Eq. (5) by choosing

$k_{x}(t)=k_{x 0}-k_{y} S t$.

This leads to a set of ordinary differential equations,

$\frac{\mathrm{d} \hat{\boldsymbol{q}}}{\mathrm{d} t}=\hat{\mathbf{L}} \hat{\boldsymbol{q}}$,

where $\hat{\mathbf{L}}$ is a matrix with coefficients that are independent of $\boldsymbol{x}$ and depend at most only on $t$.

In practice, we solve Eq. (8) numerically and monitor the evolution of the norm, $\langle\hat{\boldsymbol{q}} \mid \hat{\boldsymbol{q}}\rangle$, and of the Rayleigh quotient

$\lambda(t)=\frac{\langle\hat{\boldsymbol{q}} \mid \hat{\mathbf{L}} \hat{\boldsymbol{q}}\rangle}{\langle\hat{\boldsymbol{q}} \mid \hat{\boldsymbol{q}}\rangle}$,

where $\langle\boldsymbol{a} \mid \boldsymbol{b}\rangle=\sum_{i=1}^{N} a_{i}^{*} b_{i}$ defines a scalar product, and $N$ is the rank of the matrix $\hat{\mathbf{L}}$. We recall that, if the matrix was independent of $t$, the Rayleigh quotient would be between the largest and the smallest eigenvalue of $\hat{\mathbf{L}}$.

In the following we discuss first the MRI and turn then to the case with stratification and address the possibility of the strato-rotational instability (SRI).

\section{MRI}

\subsection{Basic equations}

Stratification is unimportant for the MRI, so we focus on the simple case with uniform background density. To simplify the problem further, we assume an isothermal equation of state, so the pressure is given by $p=c_{\mathrm{s}}^{2} \rho$. Here, $c_{\mathrm{s}}$ is the sound speed which is assumed constant. The full set of equations, in the presence of rotation with angular velocity $\mathbf{\Omega}$, is then

$\rho \frac{\mathrm{D} \boldsymbol{U}}{\mathrm{D} t}=-c_{\mathrm{s}}^{2} \boldsymbol{\nabla} \rho-2 \boldsymbol{\Omega} \times \rho \boldsymbol{U}-\rho \boldsymbol{\nabla} \psi+\boldsymbol{J} \times \boldsymbol{B}$,

$\frac{\mathrm{D} \boldsymbol{B}}{\mathrm{D} t}=\boldsymbol{B} \cdot \boldsymbol{\nabla} \boldsymbol{U}-\boldsymbol{B} \boldsymbol{\nabla} \cdot \boldsymbol{U}$

$\frac{\mathrm{D} \rho}{\mathrm{D} t}=-\rho \boldsymbol{\nabla} \cdot \boldsymbol{U}$ 
where $\mathrm{D} / \mathrm{D} t=\partial / \partial t+\boldsymbol{U} \cdot \boldsymbol{\nabla}$ is the advective derivative with respect to the total flow velocity, $\boldsymbol{B}$ is the magnetic field, $\boldsymbol{J}=\boldsymbol{\nabla} \times \boldsymbol{B} / \mu_{0}$ is the current density, and $\mu_{0}$ is the vacuum permeability.

In an accretion disc, $\psi=\frac{1}{2} \Omega^{2}\left(3 x^{2}-z^{2}\right)$ is the tidal potential that is derived by linearizing the gravitational potential with respect to some point in the midplane of the disc some distance away from the central object. However, in the following we ignore vertical gravity and assume a more general body force giving rise to the shear flow, so we assume $\psi=\Omega S x^{2}$. The equilibrium solution is then given by $\overline{\boldsymbol{U}}=(0, S x, 0), \overline{\boldsymbol{B}}=$ const, and $\rho=$ const. The mean flow is obtained by balancing $2 \boldsymbol{\Omega} \times$ $\overline{\boldsymbol{U}}$ against $\boldsymbol{\nabla} \psi$. Inserting Eq. (2) into the $\boldsymbol{U} \cdot \boldsymbol{\nabla} \boldsymbol{U}$ nonlinearity of the momentum equation, we obtain

$\boldsymbol{U} \cdot \boldsymbol{\nabla} \boldsymbol{U}=\overline{\boldsymbol{U}} \cdot \boldsymbol{\nabla} \boldsymbol{u}+\boldsymbol{u} \cdot \boldsymbol{\nabla} \overline{\boldsymbol{U}}+\boldsymbol{u} \cdot \boldsymbol{\nabla u}$,

where $\overline{\boldsymbol{U}} \cdot \boldsymbol{\nabla} \overline{\boldsymbol{U}}=0$ has been used. Note also that the term $\boldsymbol{u} \cdot \boldsymbol{\nabla} \overline{\boldsymbol{U}}=\left(0, S u_{x}, 0\right)$ can be combined with the Coriolis force $-2 \boldsymbol{\Omega} \times \boldsymbol{u}$ to give the force

$\boldsymbol{f}(\boldsymbol{u})=\left(\begin{array}{c}2 \Omega u_{y} \\ -(2 \Omega+S) u_{x} \\ 0\end{array}\right)$

which describes epicyclic deviations from purely circular motion. In terms of the departures from the mean flow, $\boldsymbol{u}$, the momentum Eq. (10) can then be written as (Brandenburg et al. 1995)

$\rho \frac{\mathrm{D} \boldsymbol{u}}{\mathrm{D} t}=\rho \boldsymbol{f}(\boldsymbol{u})-c_{\mathrm{s}}^{2} \boldsymbol{\nabla} \rho+\boldsymbol{J} \times \boldsymbol{B}$.

Likewise, in the induction equation, there is the stretching term $\boldsymbol{B} \cdot \boldsymbol{\nabla} \boldsymbol{U}$ on the right hand side of the induction Eq. (11), which leads to a term $\boldsymbol{B} \cdot \boldsymbol{\nabla} \overline{\boldsymbol{U}}=\left(0, S B_{x}, 0\right)$. We emphasize again that in the two expressions $\left(0, S u_{x}, 0\right)$ and $\left(0, S b_{x}, 0\right)$, shear only introduces terms with constant coefficients.

So far, all equations have been fully nonlinear. We can now linearize the equations about $\overline{\boldsymbol{u}}=0, \overline{\boldsymbol{B}}=$ const and denote the departures from the equilibrium solution by a prime. The linearized Lorentz force can be written in the form

$\left(\boldsymbol{\nabla} \times \boldsymbol{B}^{\prime}\right) \times \overline{\boldsymbol{B}}=\overline{\boldsymbol{B}} \cdot \boldsymbol{\nabla} \boldsymbol{B}^{\prime}-\nabla\left(\overline{\boldsymbol{B}} \cdot \boldsymbol{B}^{\prime}\right)$.

In the following two subsections we consider the cases of imposed fields that point either in the vertical or in the azimuthal directions. The former case is particularly instructive, because it allows an instability already in the much simpler axisymmetric case.

\subsection{Vertical field and axisymmetric perturbations}

For a vertical field, $\overline{\boldsymbol{B}}=\left(0,0, \bar{B}_{z}\right)$, we have

$\overline{\boldsymbol{B}} \cdot \boldsymbol{\nabla} \boldsymbol{B}^{\prime}-\boldsymbol{\nabla}\left(\overline{\boldsymbol{B}} \cdot \boldsymbol{B}^{\prime}\right)=\bar{B}_{z}\left(\begin{array}{c}\partial_{z} B_{x}^{\prime}-\partial_{x} B_{z}^{\prime} \\ \partial_{z} B_{y}^{\prime}-\partial_{y} B_{z}^{\prime} \\ 0\end{array}\right)$,

and the terms on the right hand side of the linearized induction equation are

$\overline{\boldsymbol{B}} \cdot \boldsymbol{\nabla} \boldsymbol{u}^{\prime}-\overline{\boldsymbol{B}} \boldsymbol{\nabla} \cdot \boldsymbol{u}^{\prime}=\bar{B}_{z}\left(\begin{array}{c}\partial_{z} u_{x}^{\prime} \\ \partial_{z} u_{y}^{\prime} \\ -\partial_{x} u_{x}^{\prime}-\partial_{y} u_{y}^{\prime}\end{array}\right)$.

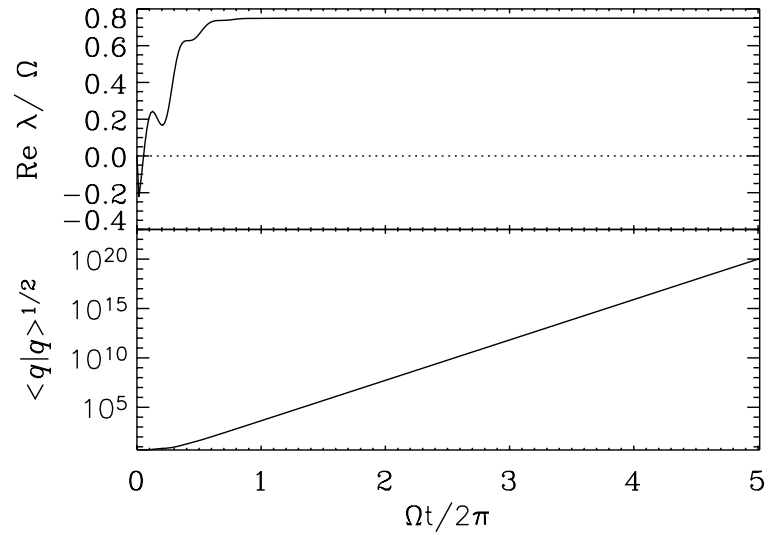

Fig. 1. Axisymmetric MRI: evolution of the real part of $\lambda$ (upper panel) and the norm $\langle q \mid q\rangle^{1 / 2}$ (or gain; see lower panel), for $v_{\mathrm{A}} k_{z}=1$ using a vertical initial field pointing in the $z$ direction, with $k_{x}=k_{y}=0$ and $S=-\frac{3}{2} \Omega$.

With these preparations we can write down the matrix $\hat{\mathbf{L}}^{(z)}$ for the MRI with an imposed vertical equilibrium field,

$\hat{\mathbf{L}}^{(z)}=\left(\begin{array}{ccccccc}0 & 2 \Omega & 0 & \mathrm{i} k_{z}^{\mathrm{A}} & 0 & -\mathrm{i} k_{x}^{\mathrm{A}} & -\mathrm{i} k_{x}^{\mathrm{c}} \\ -2 \Omega^{\mathrm{S}} & 0 & 0 & 0 & \mathrm{i} k_{z}^{\mathrm{A}} & -\mathrm{i} k_{y}^{\mathrm{A}} & -k_{y}^{\mathrm{c}} \\ 0 & 0 & 0 & 0 & 0 & 0 & -\mathrm{i} k_{z}^{\mathrm{c}} \\ \mathrm{i} k_{z}^{\mathrm{A}} & 0 & 0 & 0 & 0 & 0 & 0 \\ 0 & \mathrm{i} k_{z}^{\mathrm{A}} & 0 & S & 0 & 0 & 0 \\ -\mathrm{i} k_{x}^{\mathrm{A}} & -\mathrm{i} k_{y}^{\mathrm{A}} & 0 & 0 & 0 & 0 & 0 \\ -\mathrm{i} k_{x}^{\mathrm{c}} & -\mathrm{i} k_{y}^{\mathrm{c}} & -\mathrm{i} k_{z}^{\mathrm{c}} & 0 & 0 & 0 & 0\end{array}\right)$,

where we have used the abbreviations $2 \Omega^{\mathrm{S}}=2 \Omega+S, k_{i}^{\mathrm{A}}=k_{i} v_{\mathrm{A}}$ (sometimes also $\boldsymbol{k}_{\mathrm{A}}$ ), $k_{i}^{\mathrm{c}}=k_{i} c_{\mathrm{s}}$ (or $\boldsymbol{k}_{\mathrm{c}}$ ), and the state vector is $\hat{\boldsymbol{q}}=\left(\hat{v}_{x}, \hat{v}_{y}, \hat{v}_{z}, \hat{b}_{x}, \hat{b}_{y}, \hat{b}_{z}, \hat{\Lambda}\right)^{T}$, where the hats denote the shearing sheet expansion analogously to Eq. (6). Here we have used the rescaled variables $\Lambda=c_{\mathrm{s}} \ln \rho$ and $\boldsymbol{b}=\boldsymbol{B} / \sqrt{\mu_{0} \rho_{0}}$ to make $\hat{\mathbf{L}}$ hermitian if $S=0$. To ensure solenoidality of the magnetic field, we calculate $\hat{b}_{x}$ for the initial perturbation from (Balbus \& Hawley 1992a)

$\hat{b}_{x}=-\left(k_{y} \hat{b}_{y}+k_{z} \hat{b}_{z}\right) / k_{x 0} \quad\left(\right.$ for $\left.k_{x 0} \neq 0\right)$.

For $k_{x}=k_{y}=0$, the matrix $\hat{\mathbf{L}}$ has an unstable eigenvalue $\lambda=$ $\frac{1}{2} S$ at $k_{z}=\frac{15}{16} \Omega$. In Fig. 1 we present a solution of the ordinary differential Eq. (8) using $\hat{\mathbf{L}}^{(z)}$ for $S=-\frac{3}{2} \Omega$ and $v_{\mathrm{A}} k_{z}=1$. Note that $\operatorname{Re}(\lambda / \Omega)$ approaches $\frac{3}{4}$, i.e. the most unstable eigenvalue. This suggests that the Rayleigh quotient $\lambda(t)$ is indeed a good approximation to the most unstable eigenvalue. In agreement with earlier work (Balbus \& Hawley 1992a; Kim \& Ostriker 2000), the maximum growth rate agrees with the Oort $A$-value (Balbus \& Hawley 1992b), which is $-\frac{1}{2} S$, or $\frac{3}{4} \Omega$ for keplerian rotation.

In the special case $k_{y}=0$ (axisymmetry), $\hat{\mathbf{L}}^{(z)}$ is independent of $t$ and the solution is given by

$\hat{\boldsymbol{q}}=\tilde{\boldsymbol{q}} \mathrm{e}^{\lambda t} \quad\left(\right.$ for $\left.k_{y}=0\right)$.

This leads to the well-known dispersion relation (Balbus \& Hawley 1991)

$\left[\lambda^{4}+\lambda^{2}\left(2 k_{\mathrm{A}}^{2}+\kappa^{2}\right)+k_{\mathrm{A}}^{2}\left(k_{\mathrm{A}}^{2}-2 \Omega S\right)\right]\left(\lambda^{2}+k_{\mathrm{c}}^{2}\right)=0$, 


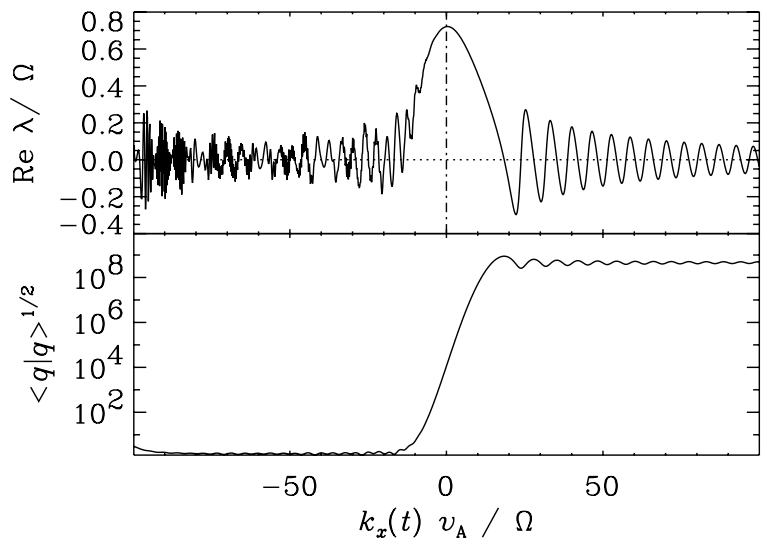

Fig. 2. Nonaxisymmetric MRI: evolution of the real part of $\lambda$ (upper panel) and the norm $\langle q \mid q\rangle^{1 / 2}$ (lower panel), for $v_{\mathrm{A}} k_{y} / \Omega=1$ using an azimuthal initial field pointing in the $y$ direction, and $k_{z}=0$.

where $\kappa^{2}=4 \Omega \Omega^{S}$ is the square of the epicyclic frequency. The resulting growth rates are

$\lambda_{ \pm}^{2}=-v_{\mathrm{A}}^{2} \boldsymbol{k}^{2}-\frac{1}{2} \kappa^{2} \pm \sqrt{4 v_{\mathrm{A}}^{2} \boldsymbol{k}^{2} \boldsymbol{\Omega}^{2}+\frac{1}{4} \kappa^{4}}$.

In the range $0<v_{\mathrm{A}}^{2} \boldsymbol{k}^{2}<2 \Omega S, \lambda$ can have real values, where $\lambda_{+}>0$, corresponding to instability.

\subsection{Azimuthal field, nonaxisymmetric perturbations}

Next we turn to the nonaxisymmetric problem. Of particular interest is the case of a purely azimuthal field $\overline{\boldsymbol{B}}=\left(0, \bar{B}_{y}, 0\right)$, which gives rise to the matrix

$$
\hat{\mathbf{L}}^{(y)}=\left(\begin{array}{ccccccc}
0 & 2 \Omega & 0 & \mathrm{i} k_{y}^{\mathrm{A}} & -\mathrm{i} k_{x}^{\mathrm{A}} & 0 & -\mathrm{i} k_{x}^{\mathrm{c}} \\
-2 \Omega^{\mathrm{S}} & 0 & 0 & 0 & 0 & 0 & -k_{y}^{\mathrm{c}} \\
0 & 0 & 0 & 0 & -\mathrm{i} k_{z}^{\mathrm{A}} & \mathrm{i} k_{y}^{\mathrm{A}} & -\mathrm{i} k_{z}^{\mathrm{c}} \\
\mathrm{i} k_{y}^{\mathrm{A}} & 0 & 0 & 0 & 0 & 0 & 0 \\
-\mathrm{i} k_{x}^{\mathrm{A}} & 0 & -\mathrm{i} k_{z}^{\mathrm{A}} & S & 0 & 0 & 0 \\
0 & 0 & \mathrm{i} k_{y}^{\mathrm{A}} & 0 & 0 & 0 & 0 \\
-\mathrm{i} k_{x}^{\mathrm{c}} & -\mathrm{i} k_{y}^{\mathrm{c}} & -\mathrm{i} k_{z}^{\mathrm{c}} & 0 & 0 & 0 & 0
\end{array}\right) .
$$

Since $\hat{\mathbf{L}}^{(y)}$ is time dependent, the solution $\hat{\boldsymbol{q}}$ will not have an exponential time dependence and the eigenvalues of $\hat{\mathbf{L}}^{(y)}$ cannot be interpreted as growth rate. Therefore we use the Rayleigh quotient $\lambda$ instead.

In Fig. 2 we show the evolution of $\operatorname{Re} \lambda$ as a function of time, except that time is translated into a corresponding variation of $k_{x}(t)$; see Eq. (7). In the second panel we show the corresponding variation of $\langle\boldsymbol{q} \mid \boldsymbol{q}\rangle^{1 / 2}$, where we see an increase over about 8 orders of magnitude during the time interval in which $\operatorname{Re} \lambda$ is systematically positive. Note also that $\max (\operatorname{Re} \lambda) \approx \frac{3}{4} \Omega$ (upper panel of Fig. 2), which is indeed the maximum growth rate of the axisymmetric MRI. This supports our interpretation that the Rayleigh quotient provides a convenient and quantitative means of estimating the growth rate of the instability.

In Fig. 3 we show a two-dimensional parameter survey in the $(k, S)$ plane of the gain factor and of $\max (\operatorname{Re} \lambda)$. Note that both the gain factor and $\max (\operatorname{Re} \lambda)$ increase toward more negative values of $S$. Remarkable is the fact that on the Rayleigh

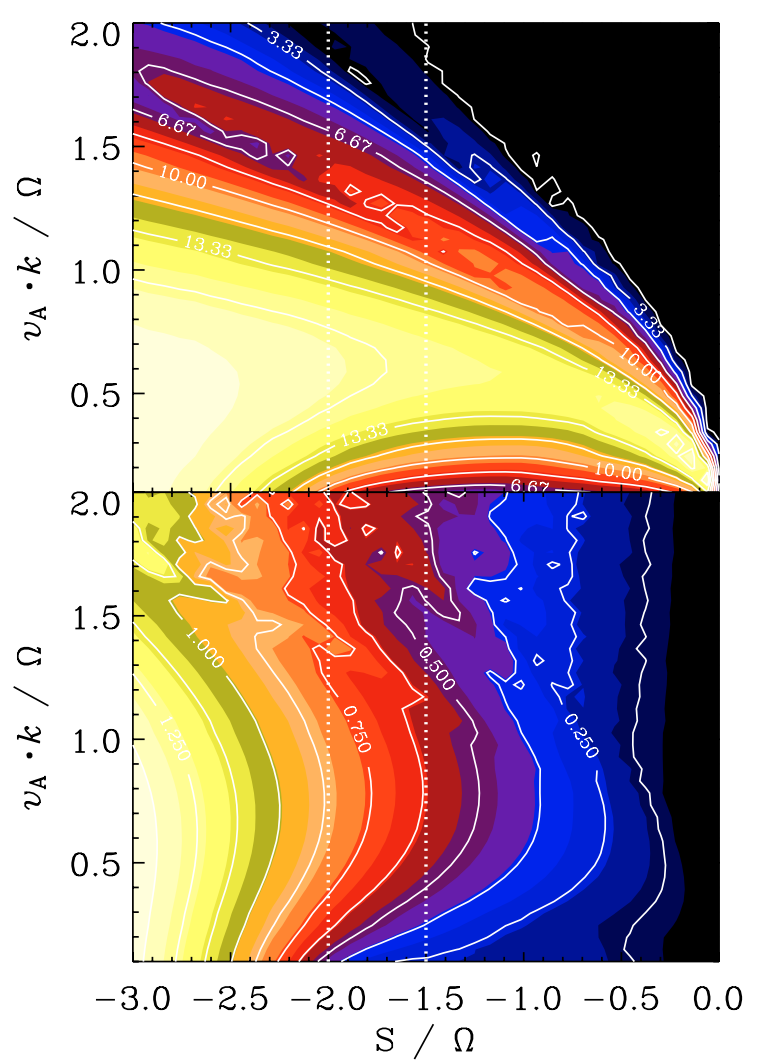

Fig. 3. Nonaxisymmetric MRI: dependence of the gain factor in logarithmic representation (upper panel) and of $\max _{t}(\operatorname{Re} \lambda) / \Omega$ (lower panel) on $S / \Omega$ and $v_{\mathrm{A}} k / \Omega$ for a vertical field. Note that $\max _{t}(\operatorname{Re} \lambda) / \Omega$ reaches the value of $\frac{1}{2} S / \Omega$ for different values of $S / \Omega$.

line, $S / \Omega=-2$, both quantities vary smoothly and do not show any special behavior.

\subsection{Direct numerical verification}

It is instructive to compare the present modal analysis with a direct three-dimensional simulation of the fully nonlinear equations in real space. This is done in Fig. 4 where we compare the increase of $\langle\boldsymbol{q} \mid \boldsymbol{q}\rangle^{1 / 2}$ with the resulting evolution of the rootmean-square magnetic field from a direct simulation of the shearing sheet equations. Here we also adopt an isothermal gas with constant sound speed.

The initial condition for the 3-dimensional direct simulation is obtained by evolving linearized shearing sheet equations for $k_{y}=1$ and $k_{z}=10$ to the point where $k_{x}\left(t_{0}\right)=-5$. [The size of the domain is $(2 \pi)^{3}$.] For definitiveness, we reproduce here the numerical values in Eq. (6):

$\hat{\boldsymbol{u}}=\left(\begin{array}{c}-0.311-0.037 \mathrm{i} \\ -0.461-0.054 \mathrm{i} \\ -0.088-0.010 \mathrm{i}\end{array}\right), \quad \hat{\boldsymbol{b}}=\left(\begin{array}{c}+0.068-0.581 \mathrm{i} \\ -0.039+0.334 \mathrm{i} \\ +0.035-0.295 \mathrm{i}\end{array}\right)$

and the logarithmic density is given by $\hat{\Lambda}=0.042-0.3647 \mathrm{i}$. The amplitude is chosen to be $A=10^{-4}$. Figure 5 shows images of $B_{z}$ on the periphery of the simulation domain at different 


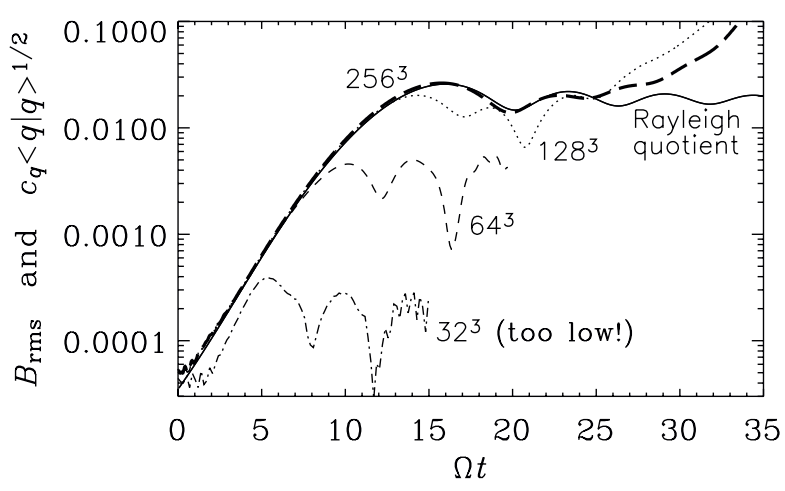

Fig. 4. Transient amplification of the magnetic field by the nonaxisymmetric MRI with an azimuthal field. The solid line shows the result from the Rayleigh quotient method while the broken lines give the result from direct three-dimensional simulations with zero viscosity and zero resistivity. The square root of the Rayleigh quotient has been scaled by a factor $c_{q}=3.5 \times 10^{-5}$ to make it overlap with $B_{\mathrm{rms}}$ curve. A resolution of only $32^{3}$ meshpoints is completely insufficient to resolve even the beginning of the instability. At least $256^{3}$ meshpoints are required to resolve the maximum (thick dashed line). After $\Omega t>17$ even the simulation with $256^{3}$ meshpoints becomes under-resolved.

times ${ }^{1}$. The simulations have been carried out using the PENCIL $\mathrm{CODE}^{2}$ which is a high-order finite-difference code (sixth order in space and third order in time) for solving the compressible hydromagnetic equations.

The way how this transient amplification can lead to sustained growth is through mode coupling, which is not considered in the present analysis. Relevant mode couplings could come about either through nonuniformities in the cross-stream or $x$ direction and through boundary conditions, or through nonlinearities. In the shearing sheet approximation only the latter is a viable possibility, and this is probably the mechanism through which the early shearing sheet simulations produced sustained turbulence (e.g. Hawley et al. 1995; Matsumoto \& Tajima 1995; Brandenburg et al. 1995).

We conclude this section by emphasizing the one-to-one correspondence between numerical solutions of the shearing sheet equations and the Rayleigh quotient method. The nonaxisymmetric case is similar to the axisymmetric one in that the maximum growth rate is the same. This is achieved for $k_{x}=0$, which, in the nonaxisymmetric case, can only be the case at one instance in time.

\section{Shear flow with stratification}

We now turn to the case with vertical stratification. In order to allow for gravity waves, we need to abandon the isothermal equation of state and use instead a perfect gas law. In terms of specific entropy, $s$, we can formulate this as

$s=c_{v} \ln p-c_{p} \ln \rho+s_{0}$,

\footnotetext{
1 Animations of the flow can be found at http://www.nordita.dk/brandenb/movies/disc and at http://www. edpsciences.org

${ }^{2}$ http://www. nordita.dk/software/pencil-code
}

where $c_{p}$ and $c_{v}$ are the specific heats at constant pressure and constant volume, respectively. The specific entropy is only defined up to an additive constant, $s_{0}$. In the absence of heating and cooling, the specific entropy remains constant on streamlines, i.e.

$\frac{\mathrm{D} s}{\mathrm{D} t}=0$.

Using Eqs. (24) and (25) we can rewrite the continuity Eq. (12) in the form

$\frac{\mathrm{D} p}{\mathrm{D} t}=-\gamma p \boldsymbol{\nabla} \cdot \boldsymbol{u}$,

where $\gamma=c_{p} / c_{v}$ is the ratio of specific heats and $\boldsymbol{\nabla} \cdot \overline{\boldsymbol{U}}=0$ has been used. We solve Eqs. (25) and (26) together with the momentum equation in the form

$\rho \frac{\mathrm{D} \boldsymbol{u}}{\mathrm{D} t}=\rho \boldsymbol{f}(\boldsymbol{u})-\boldsymbol{\nabla} p+\rho \boldsymbol{g}$

where $\boldsymbol{f}(\boldsymbol{u})$ has been defined in Eq. (14). These equations have an isothermal equilibrium solution (denoted by an overbar),

$\overline{\boldsymbol{u}}=\mathbf{0}, \quad \bar{s}(z)=\frac{c_{p}-c_{v}}{H}, \quad \bar{p}(z)=p_{0} \mathrm{e}^{-z / H}$,

where

$H=c_{\mathrm{s}}^{2} /(\gamma g)$

is the pressure scale height and $c_{\mathrm{s}}$ is the sound speed. Both $H$ and $c_{\mathrm{s}}$ are constants, and so is $p_{0}$ which gives the pressure at $z=0$. The equilibrium density is given by $\bar{\rho}=\gamma \bar{p} / c_{\mathrm{s}}^{2}$.

Linearizing Eqs. (24)-(27) about the $z$ dependent equilibrium solution yields

$\frac{s^{\prime}}{c_{p}}=\frac{p^{\prime}}{\gamma \bar{p}}-\frac{\rho^{\prime}}{\bar{\rho}}$

$\frac{\partial s^{\prime}}{\partial t}+u_{z}^{\prime} \frac{\mathrm{d} \bar{s}}{\mathrm{~d} z}=0$,

$\frac{\partial p^{\prime}}{\partial t}+u_{z}^{\prime} \frac{\mathrm{d} \bar{p}}{\mathrm{~d} z}=-\gamma \bar{p} \boldsymbol{\nabla} \cdot \boldsymbol{u}^{\prime}$,

$\bar{\rho}(z) \frac{\partial \boldsymbol{u}^{\prime}}{\partial t}=\bar{\rho}(z) \boldsymbol{f}\left(\boldsymbol{u}^{\prime}\right)-\boldsymbol{\nabla} p^{\prime}+\rho^{\prime} \boldsymbol{g}$,

where primes denote deviations from the equilibrium. These four equations have non-constant coefficients. Using rescaled variables (denoted by a tilde),

$s^{\prime}=\sqrt{\gamma-1} c_{p} \tilde{s} \mathrm{e}^{z / 2 H}$,

$p^{\prime}=\gamma p_{0} \tilde{p} \mathrm{e}^{-z / 2 H}$,

$\boldsymbol{u}^{\prime}=c_{\mathrm{s}} \tilde{\boldsymbol{u}} \mathrm{e}^{z / 2 H}$,

and eliminating $\rho^{\prime}$ using Eq. (30), we can rewrite these equations in the form

$\frac{\partial \tilde{s}}{\partial t}=-\sqrt{\gamma-1} \frac{c_{\mathrm{s}}}{\gamma H} \tilde{u}_{z}$ 

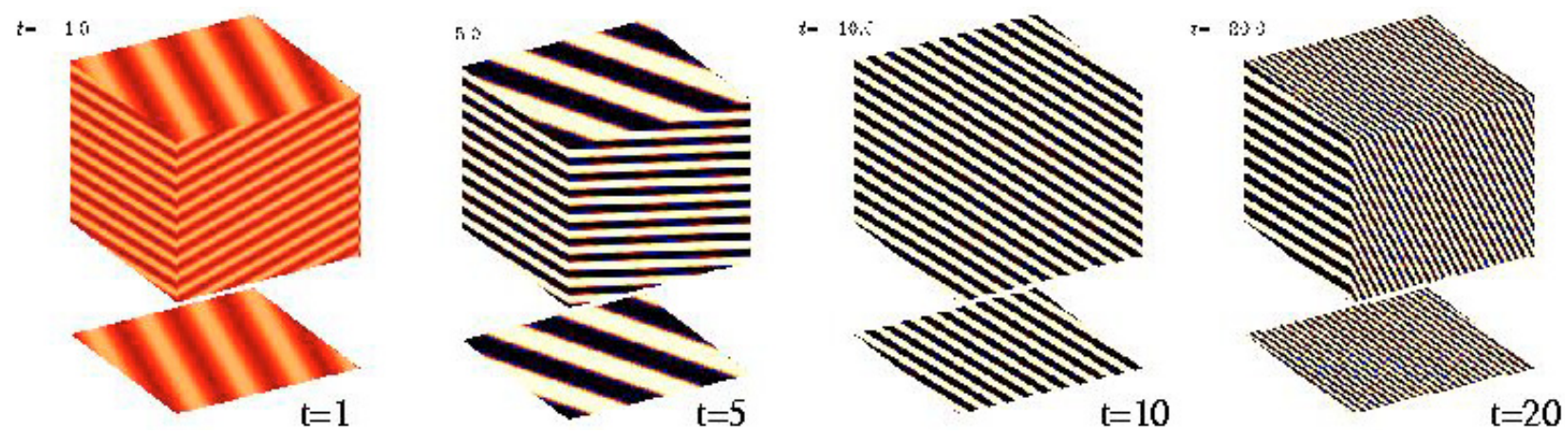

Fig. 5. Images of the vertical component of the magnetic field, $B_{z}$, for the nonaxisymmetric MRI with an azimuthal field, using different values of $t$, where the field at $t=0$ corresponds to that given by Eq. (23).

$\frac{\partial \tilde{p}}{\partial t}=\frac{c_{\mathrm{s}}}{\gamma H} \tilde{u}_{z}-c_{\mathrm{s}}\left(\frac{\tilde{u}_{z}}{2 H}+\nabla \cdot \tilde{\boldsymbol{u}}\right)$

$\frac{\partial \tilde{\boldsymbol{u}}}{\partial t}=\boldsymbol{f}(\tilde{\boldsymbol{u}})+c_{\mathrm{S}}\left(\frac{\hat{z}}{2 H}-\nabla\right) \tilde{p}+(\tilde{p}-\sqrt{\gamma-1} \tilde{s}) \frac{\boldsymbol{g}}{c_{\mathrm{S}}}$.

It is now convenient to introduce nondimensional time and space coordinates. A natural length scale to choose might be $H$, or better $\gamma H=c_{\mathrm{s}}^{2} / g$. The problem with this is that the unstratified limit $g \rightarrow 0$ is then ill-posed, because $H \rightarrow \infty$. A more flexible alternative is therefore to choose a "system" scale $L$, or better $\gamma L$, as our length scale. (The length scale $L$ is not to be confused with the matrix $\mathbf{L}$.) Thus, we define nondimensional inverse time and length scales via

$\frac{\partial}{\partial t}=\frac{c_{\mathrm{s}}}{\gamma L} \frac{\partial}{\partial \tilde{t}} \quad$ and $\quad \boldsymbol{\nabla}=\frac{1}{\gamma L} \tilde{\nabla}$

The final set of equations is then

$\frac{\partial \tilde{s}}{\partial \tilde{t}}=-\frac{L}{H} \sqrt{\gamma-1} \tilde{u}_{z}$,

$\frac{\partial \tilde{p}}{\partial \tilde{t}}=\frac{L}{H}\left(1-\frac{\gamma}{2}\right) \tilde{u}_{z}-\tilde{\nabla} \cdot \tilde{\boldsymbol{u}}$,

$\frac{\partial \tilde{\boldsymbol{u}}}{\partial \tilde{t}}=\tilde{\boldsymbol{f}}(\tilde{\boldsymbol{u}})-\frac{L}{H}\left(1-\frac{\gamma}{2}\right) \hat{z} \tilde{p}-\tilde{\nabla} \tilde{p}+\frac{L}{H} \sqrt{\gamma-1} \hat{z} \tilde{s}$,

where we have defined $\tilde{\boldsymbol{f}}(\tilde{\boldsymbol{u}})=\left(2 \tilde{\Omega} u_{y},-2 \tilde{\Omega}^{S} u_{x}, 0\right)^{T}$ with $2 \tilde{\Omega}^{S}=$ $2 \tilde{\Omega}+\tilde{S}$. Here, $\tilde{\Omega}=\gamma L \Omega / c_{\mathrm{s}}$ and $\tilde{S}=\gamma L S / c_{\mathrm{S}}$ are nondimensional angular and shear velocities. We recall that $\boldsymbol{g}=(0,0,-g)$. As a nondimensional measure for the degree of stratification we use in the following the symbol

$\tilde{g}=\frac{\gamma L g}{c_{\mathrm{s}}^{2}} \equiv \frac{L}{H}$.

The case $\tilde{g}=0$ corresponds obviously to the completely unstratified case.

Next, we write these equations in matrix form (5), make the ansatz (6), and have a set of ordinary differential Eqs. (8) with the matrix $\hat{\mathbf{L}}$ in the form

$\hat{\mathbf{L}}=\left(\begin{array}{ccccc}0 & 2 \tilde{\Omega} & 0 & 0 & -\mathrm{i} k_{x}(t) \\ -2 \tilde{\Omega}^{S} & 0 & 0 & 0 & -\mathrm{i} k_{y} \\ 0 & 0 & 0 & \tilde{N} & -\tilde{M}-\mathrm{i} k_{z} \\ 0 & 0 & -\tilde{N} & 0 & 0 \\ -\mathrm{i} k_{x}(t) & -\mathrm{i} k_{y} & \tilde{M}-\mathrm{i} k_{z} & 0 & 0\end{array}\right)$, operating on the state vector $\hat{\boldsymbol{q}}=\left(\hat{u}_{x}, \hat{u}_{y}, \hat{u}_{z}, \hat{s}, \hat{p}\right)^{T}$, which is defined according to Eq. (6),

$\tilde{N}=\frac{L}{H} \sqrt{\gamma-1}$

is the nondimensional Brunt-Väisälä frequency, and

$\tilde{M}=\frac{L}{H}\left(1-\frac{\gamma}{2}\right)$

is another nondimensional number characterizing the degree of stratification. Note that in the absence of shear, $S=0$, the matrix $\hat{\mathbf{L}}$ is skew-hermitian, i.e. $\hat{\mathbf{L}}_{i j}=-\hat{\mathrm{L}}_{j i}^{*}$, and all eigenvalues are purely imaginary or zero. A similar nondimensionalization was used by Brandenburg (1988) for the case $\Omega=0$, but space and time coordinates were scaled such that the coefficient $\tilde{M}$ became unity. As discussed above, this gives an unnecessary restriction in that it prevents us from making a continuous transition to the unstratified case $L / H=0$. Furthermore, in the special case $\gamma=2$, we have $\tilde{M}=0, \tilde{N}=L / H \neq 0$, which corresponds to the Boussinesq case if we also make the assumption of incompressibility. Technically, the latter is achieved by multiplying the left hand side of Eq. (8) by the matrix $\operatorname{diag}(1,1,1,1,0)$. This means that $\tilde{p}$ can be expressed in terms of $\tilde{\boldsymbol{u}}$, and we are left with only 4 explicitly timedependent equations.

In the axisymmetric case, $k_{y}=0$, or in the absence of shear, $S=0$, the eigenvalues $\lambda$ satisfy the dispersion relation $\lambda D(\lambda, \boldsymbol{k})=0$ with

$D=\lambda^{4}+\lambda^{2}\left(k^{2}+\kappa^{2}+\tilde{K}^{2}\right)+k_{x}^{2} \tilde{N}^{2}+\kappa^{2}\left(k_{z}^{2}+\tilde{K}^{2}\right)$,

where $k^{2}=k_{x}^{2}+k_{z}^{2}, \kappa$ is the nondimensional epicyclic frequency with $\kappa^{2}=4 \tilde{\Omega} \tilde{\Omega}^{S}$, and $\tilde{K}^{2}=\tilde{N}^{2}+\tilde{M}^{2}$ has been introduced as an abbreviation. Written in dimensional form, this dispersion relation is identical to the usual one for atmospheric waves in an isothermally stratified atmosphere (Stein \& Leibacher 1974; Ryu \& Goodman 1992). There are five solutions, one zerofrequency mode, $\lambda=0$, and four others with $\operatorname{Im} \lambda= \pm \omega_{\mathrm{p}}(\boldsymbol{k})$ and $\operatorname{Im} \lambda= \pm \omega_{\mathrm{g}}(\boldsymbol{k})$, corresponding to $\mathrm{p}$ - and g-modes, respectively.

Only in the axisymmetric case, $k_{y}=0$, $\hat{\mathbf{L}}$ becomes independent of $t\left(k_{x}=k_{x 0}=\right.$ const) and so Eq. (48) can be used even when $S \neq 0$. From Eq. (48) it is clear that now instability requires

$\kappa^{2}<-k_{x}^{2} \tilde{N}^{2} /\left(k_{z}^{2}+\tilde{K}^{2}\right)$ 


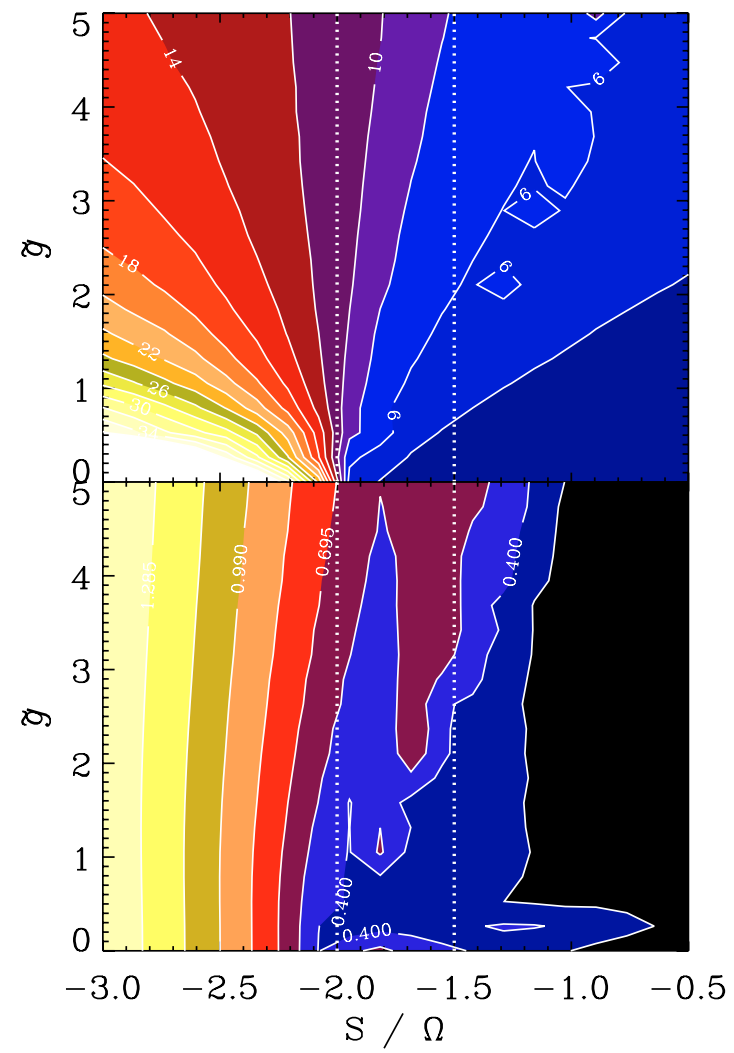

Fig. 6. Nonaxisymmetric stratified case: dependence of the gain factor in linear representation (upper panel) and $\max _{t}(\lambda) / \Omega$ (lower panel) on $S / \Omega$ and $\tilde{g}$, for $\gamma L k_{z}=1$.

which is also known as the Solberg-Høiland criterion (e.g. Rüdiger \& Shalybkov 2002; Narayan et al. 2002). Negative values of $\kappa^{2}=4 \tilde{\Omega} \tilde{\Omega}^{S}$ are possible when $-S>2 \Omega$, corresponding to Rayleigh's criterion. Instability occurs closest to the Rayleigh line when $k_{x}^{2}$ is small and $k_{z}^{2}$ large.

In Fig. 6 we present the results of a two-dimensional parameter survey varying both the degree of stratification, $\tilde{g}$ as well as the strength of the shear, $S$. The Rayleigh stability line, $S / \Omega=-2$, is particularly evident in the plot of the gain factor (upper panel): for zero stratification the gain factor increases sharply as one crosses the Rayleigh line. For stronger stratification, the increase in the gain factor diminishes, suggesting that stratification has a stabilizing influence on the Rayleigh-Taylor instability.

For $S / \Omega>-2$, the flow is known to be axisymmetrically stable. Also in the nonaxisymmetric case the gain factor is negligible. Nevertheless, in the $(\tilde{g}, S)$ parameter plane there are regions with an appreciable maximum value of $\lambda$ (lower panel of Fig. 6). However, as is seen more clearly in the corresponding time trace (Fig. 7), a positive maximum of $\lambda$ is more or less compensated by a corresponding amount of negative contributions. This explains the absence of a corresponding pattern of $\max _{t}(\lambda)$ in the $(\tilde{g}, S)$ plane. This suggests also that, in contrast to simulations of the MRI, the transients would be insignificant to produce sustained growth.

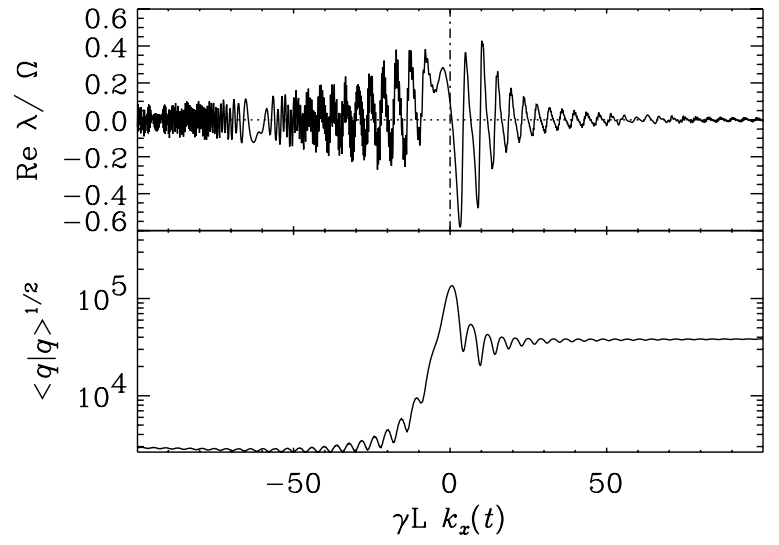

Fig. 7. Nonaxisymmetric stratified case: dependence of the gain factor and $\max _{t}(\lambda) / \Omega$ on $\gamma L k_{x}(t)$, for $\gamma L k_{z}=1$.

\section{Conclusions}

The shearing sheet approximation is a powerful tool to analyze the behavior of an accretion disc locally. While this approximation eliminates a wide class of global instabilities, it has the advantage of isolating local instabilities that are often believed to be responsible for driving turbulence. Our work has demonstrated that for sufficiently weak perturbations there is a direct correspondence between the solutions of shearing sheet equations as obtained from a numerical code and the Rayleigh quotient obtained by solving the modal equations with a time-dependent radial wavenumber $k_{x}(t)$.

Within the framework of the shearing sheet approximation we have demonstrated the stability of a stratified isothermal atmosphere with horizontal keplerian shear to fully compressible perturbations. The behavior of the solution is well characterized by the Rayleigh quotient, $\lambda(t)$, which is obtained for a range of different initial conditions. The strato-rotational instability (SRI), which has been seen in experiments and calculations with radial boundaries (Molemaker et al. 2001; Shalybkov \& Rüdiger 2005), can therefore not be a local instability, as was already discussed by Umurhan (2005) and Lesur \& Longaretti (2005).

The present work has also highlighted the absence of any correspondence between the growth rates obtained from the pseudo-dispersion relation and the actual evolution of $\operatorname{Re} \lambda(t)$. Of course, there will be transient growth from almost any initial condition and for any value of $S / \Omega$-including $S / \Omega>0$, which would be stable even in the presence of magnetic fields (Balbus \& Hawley 1991). However, under certain conditions this can lead to what is known as the bypass transition (Chagelishvili et al. 2003; Afshordi et al. 2005), i.e. the transient growth that can lead to a new state that is itself unstable. Nevertheless, the presence of stratification $(\tilde{g}>0)$ is here seen to have a stabilizing effect, making the connection with the SRI implausible.

We emphasize that in the non-Boussinesq stratified case it is important to remove $z$-dependent coefficients before making the $\exp \left(\mathrm{i} k_{z} z\right)$ ansatz. Otherwise, persistent real parts of $\lambda(t)$ will arise that reflect merely the fact that velocity increases as a wave packet travels into less dense regions. 
Finally, it should be pointed out that, if keplerian shear flows were locally unstable in stratified media, one might have seen this in the fully nonlinear shearing sheet simulations of Brandenburg et al. (1995), where the Lorentz force was removed and the dynamo-driven turbulence was found to decay rapidly; see their Fig. 4. A similar test was also done by Hawley et al. (1995), but only for the unstratified case which is not relevant here. We note that real discs have vertical shear which does allow for a hydrodynamic instability, but it is far less powerful than the Balbus-Hawley instability (e.g. Urpin \& Brandenburg 1998; Arlt \& Urpin 2004). Other proposals for hydrodynamic instability include the baroclinic instability (Klahr \& Bodenheimer 2003) that has been discussed in the context of protostellar discs.

Acknowledgements. We thank Bérengère Dubrulle for numerous email conversations on the subject of this paper. This work has been supported by the European Commission under the Marie-Curie grant HPMF-CT-1999-00411. The Danish Center for Scientific Computing is acknowledged for granting time on the Horseshoe cluster.

\section{References}

Arlt, R., \& Urpin, V. 2004, A\&A, 426, 755

Afshordi, N., Mukhopadhyay, B., \& Narayan, R. 2005, ApJ, 629, 373

Balbus, S. A., \& Hawley, J. F. 1991, ApJ, 376, 214

Balbus, S. A., \& Hawley, J. F. 1992a, ApJ, 400, 610
Balbus, S. A., \& Hawley, J. F. 1992b, ApJ, 392, 662

Balbus, S. A., \& Hawley, J. F. 1998, Rev. Mod. Phys., 70, 1

Brandenburg, A. 1988, A\&A, 203, 154

Brandenburg, A., \& Rüdiger, G. 2006, A\&A, submitted [arXiv:astro-ph/0512409]

Brandenburg, A., Nordlund, Å., Stein, R. F., \& Torkelsson, U. 1995, ApJ, 446, 741

Chagelishvili, G. D., Zahn, J.-P., Tevzadze, A. G., \& Lominadze, J. G. 2003, A\&A, 402, 401

Dubrulle, B., Marié, L., Normand, Ch., et al. 2005, A\&A, 429, 1

Frank, J., King, A. R., \& Raine, D. J. 1992, Accretion power in astrophysics (Cambridge: Cambridge Univ. Press)

Goldreich, P., \& Lynden-Bell, D. 1965, MNRAS, 130, 125

Hawley, J. F., Gammie, C. F., \& Balbus, S. A. 1995, ApJ, 440, 742

Kim, W.-T., \& Ostriker, E. C. 2000, ApJ, 540, 372

Klahr, H., \& Bodenheimer, P. 2003, ApJ, 582, 869

Lesur, G., \& Longaretti, P.-Y. 2005, A\&A, 444, 25

Matsumoto, R., \& Tajima, T. 1995, ApJ, 445, 767

Molemaker, M. J., McWilliams, J. C., \& Yavneh, I. 2001, PRL, 86, 5270

Narayan, R., Quataert, E., Igumenshchev, I. V., \& Abramowicz, M. A. 2002, ApJ, 577, 295

Ogilvie, G. I., \& Pringle, J. E. 1996, MNRAS, 279, 152

Rüdiger, G., \& Shalybkov, D. 2002, PRE, 66, 016307

Ryu, D., \& Goodman, J. 1992, ApJ, 388, 438

Shakura, N. I., \& Sunyaev, R. A. 1973, A\&A, 24, 337

Shalybkov, D., \& Rüdiger, G. 2005, A\&A, 438, 411

Stein, R. F., \& Leibacher, J. 1974, A\&AR, 12, 407

Umurhan, O. M. 2006, MNRAS, 365, 85

Urpin, V., \& Brandenburg, A. 1998, MNRAS, 294, 399 